\title{
CHIRAL SYMMETRY BREAKING FOR FUNDAMENTAL FERMIONS
}

\author{
A. Bashir \\ Centre for Particle Theory, \\ University of Durham, \\ Durham DH1 3LE, U.K.
}

\section{INTRODUCTION}

Massive fermions have long been a problem in gauge theories. Unification of electromagnetic and weak forces was once hindered by the fact that the introduction of mass terms broke the gauge invariance of the theory. This problem was solved by the introduction of the Higgs field. Spontaneous breakdown of the $\mathrm{SU}(2) \times \mathrm{U}(1)$ symmetry then takes place. The gauge bosons gain mass and the masses for the fermions are generated through their Yukawa interaction with this Higgs field. However, there has been a widespread dissatisfaction with this mechanism since the masses are not predictable. Rather, they must be fixed by experiment. Studying the non-perturbative behaviour of gauge theories provides an alternative. If the interactions are strong enough, they are capable of generating masses for the particles dynamically even if they start with zero bare mass. Moreover, experiment tells us that the top quark is very heavy and so the Yukawa coupling $g_{t}$ for top-Higgs interaction is $\mathrm{O}(1)$. Then one naturally expects that non-perturbative effects become important. Indeed, it has been suggested [1] that the top quark may acquire mass non-perturbatively through four-fermion interactions, and the Higgs can then be viewed as the condensate of the top and the antitop. However, in an attempt to include the effects of gauge boson exchange term, one loses gauge invariance of the physical quantities. Of course, physical quantities must be gauge independent. This motivates the study of how to achieve this in non-perturbative calculations. Quenched QED provides a toy model in which to study this problem, as we discuss. 


\section{DYSON-SCHWINGER EQUATIONS}

Our starting point is the set of Dyson-Schwinger equations. These are an infinite system of coupled equations for all the Green's functions, which are non-perturbative in nature. Their structure is such that the 1-point function is related to the 2-point function, the 2-point function is related to the 3-point function, etc. ad infinitum. As it is impossible to solve the complete set of equations, one has to truncate this infinite tower in a physically acceptable way to reduce them to something that is soluble. A familiar way to do this is perturbation theory. However, if one wishes to generate masses for particles, a non-perturbative way has to be sought.

To see how to do this consider two of the Dyson-Schwinger equations, one for the fermion propagator, and the other for the photon propagator. These are shown below diagrammatically together with their corresponding mathematical expressions:

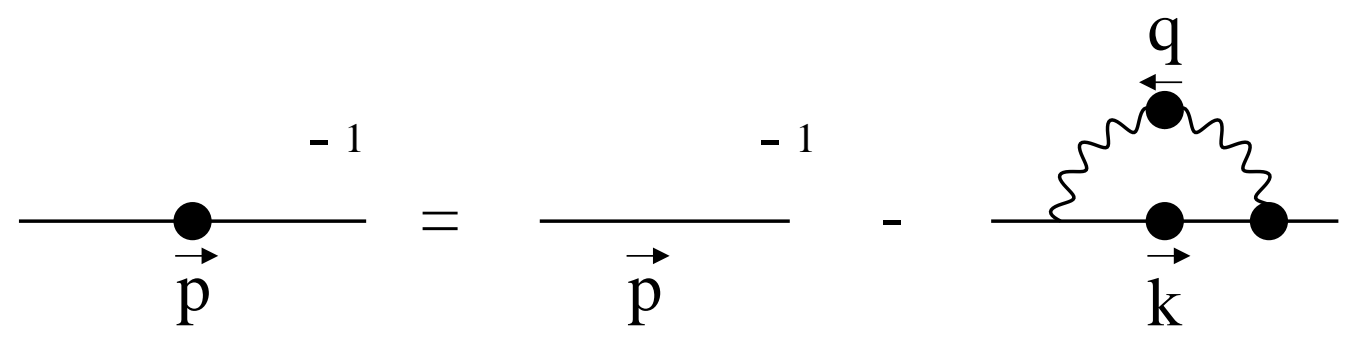

FIG. 1. Dyson-Schwinger equation for fermion propagator.

$$
i S_{F}^{-1}(p)=i S_{F}^{0^{-1}}(p)-e^{2} \int \frac{d^{4} k}{(2 \pi)^{4}} \gamma^{\mu} S_{F}(k) \Gamma^{\nu}(k, p) \Delta_{\mu \nu}(q),
$$

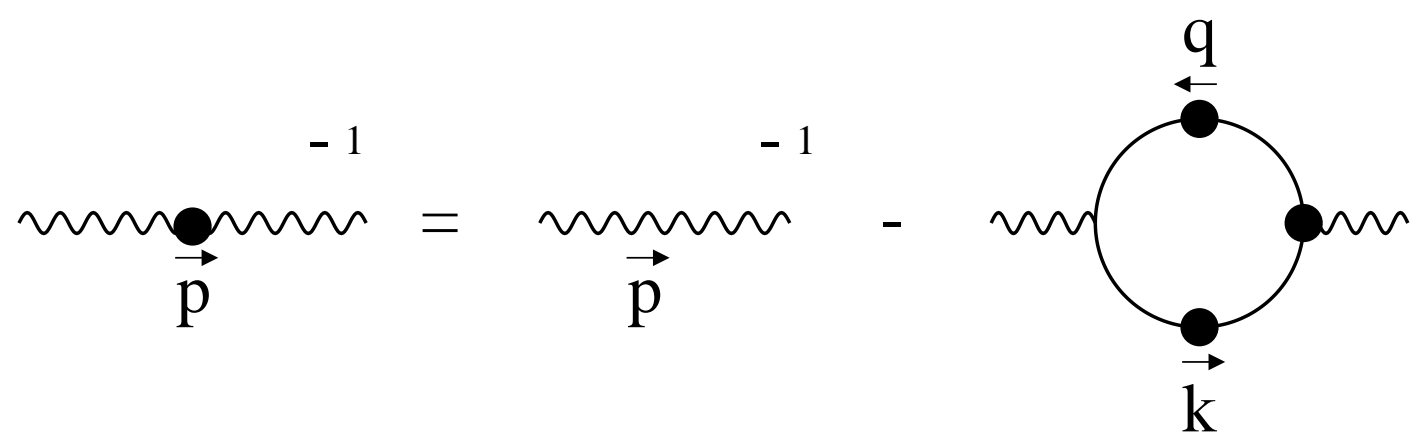

FIG. 2. Dyson-Schwinger equation for photon propagator.

$$
i \Delta_{\mu \nu}^{-1}(p)=i \Delta_{\mu \nu}^{0^{-1}}(p)-e^{2} N_{f} \int \frac{d^{4} k}{(2 \pi)^{4}} \gamma^{\mu} S_{F}(k) \Gamma^{\nu}(k, p) S_{F}(q)
$$


where the quantities with the superscript ' 0 ' are bare quantities, and the others are full ones. Quenched QED corresponds to making the assumption that the full photon propagator can be replaced by its bare counterpart. This limit is achieved by regarding $N_{f}$ as a mathematical parameter, which is set equal to zero. As an example, to begin with, we make a further simplification by replacing the full vertex by the bare one. Eq. (1) then reduces to:

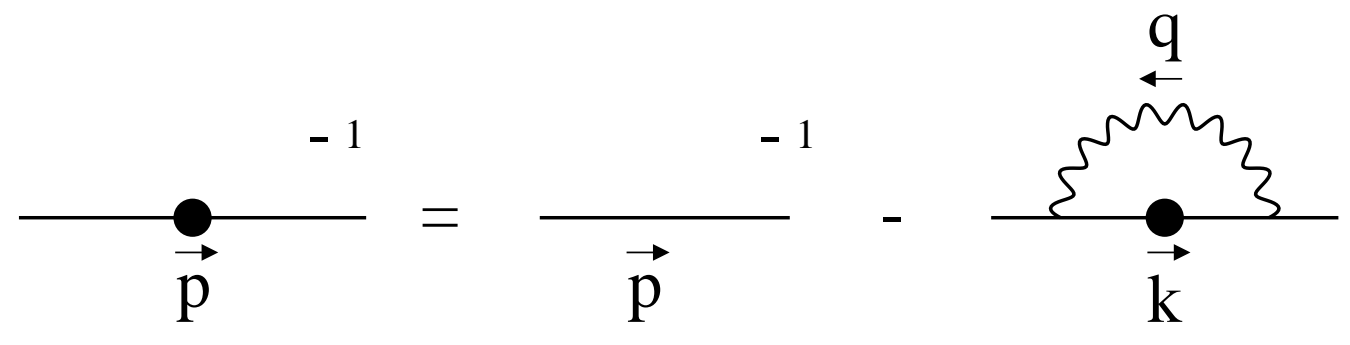

FIG. 3. Rainbow approximation.

$$
i S_{F}^{-1}(p)=i S_{F}^{0^{-1}}(p)-e^{2} \int \frac{d^{4} k}{(2 \pi)^{4}} \gamma^{\mu} S_{F}(k) \gamma^{\nu}(k, p) \Delta_{\mu \nu}^{0}(q),
$$

in what is known as the rainbow approximation, where

$$
\begin{aligned}
S_{F}(k) & =\frac{F\left(k^{2}\right)}{\not k-\mathcal{M}\left(k^{2}\right)}, \\
S_{F}^{0}(k) & =\frac{1}{\not k-m_{0}}, \\
\Delta_{\mu \nu}^{0}(q) & =\frac{1}{q^{2}}\left(g_{\mu \nu}+(\xi-1) \frac{q_{\mu} q_{\nu}}{q^{2}}\right) .
\end{aligned}
$$

Eq. (3) is a matrix equation which corresponds to two equations in $\mathcal{M}$ and $\mathrm{F}$. We can project out equations for these by taking the trace of Eq. (3) having multiplied by $\not p$ and 1 in turn to obtain:

$$
\begin{aligned}
\frac{1}{F\left(p^{2}\right)}= & 1-\frac{\alpha}{4 \pi^{3}} \frac{1}{p^{2}} \int d^{4} k \frac{F\left(k^{2}\right)}{k^{2}+\mathcal{M}^{2}\left(k^{2}\right)} \frac{1}{q^{2}} . \\
& \left\{-2 k \cdot p-\frac{(\xi-1)}{q^{2}}\left[2 k^{2} p^{2}-\left(k^{2}+p^{2}\right) k \cdot p\right]\right\}, \\
\frac{\mathcal{M}\left(p^{2}\right)}{F\left(p^{2}\right)}= & m_{0}-\frac{\alpha}{4 \pi^{3}} \int d^{4} k \frac{F\left(k^{2}\right) \mathcal{M}\left(k^{2}\right)}{k^{2}+\mathcal{M}^{2}\left(k^{2}\right)} \frac{1}{q^{2}}(3+\xi) .
\end{aligned}
$$

where as usual $\alpha=e^{2} / 4 \pi$. On carrying out the angular integrations, and putting the 
bare mass equal to zero, we have

$$
\begin{aligned}
\frac{1}{F\left(p^{2}\right)} & =1+\frac{\alpha \xi}{4 \pi} \int_{0}^{\Lambda^{2}} d k^{2} \frac{F\left(k^{2}\right)}{k^{2}+\mathcal{M}^{2}\left(k^{2}\right)}\left[\frac{k^{4}}{p^{4}} \theta\left(p^{2}-k^{2}\right)+\theta\left(k^{2}-p^{2}\right)\right], \\
\frac{\mathcal{M}\left(p^{2}\right)}{F\left(p^{2}\right)} & =\frac{\alpha(3+\xi)}{4 \pi} \int_{0}^{\Lambda^{2}} d k^{2} \frac{\mathcal{M}\left(k^{2}\right) F\left(k^{2}\right)}{k^{2}+\mathcal{M}^{2}\left(k^{2}\right)}\left[\frac{k^{2}}{p^{2}} \theta\left(p^{2}-k^{2}\right)+\theta\left(k^{2}-p^{2}\right)\right],
\end{aligned}
$$

where $\Lambda$ is the ultra-violet momentum cutoff. It is easiest to solve these equations in the Landau gauge where they decouple. $F\left(p^{2}\right)$ is obviously 1. Moreover, there is a non-trivial solution [2] for the mass function $\mathcal{M}$ for the coupling larger than a critical value of $\alpha_{c}=\pi / 3$. This is best illustrated by plotting the Euclidean mass $M=\mathcal{M}\left(M^{2}\right)$ as a function of $\alpha$, as found by Curtis and Pennington [3]:

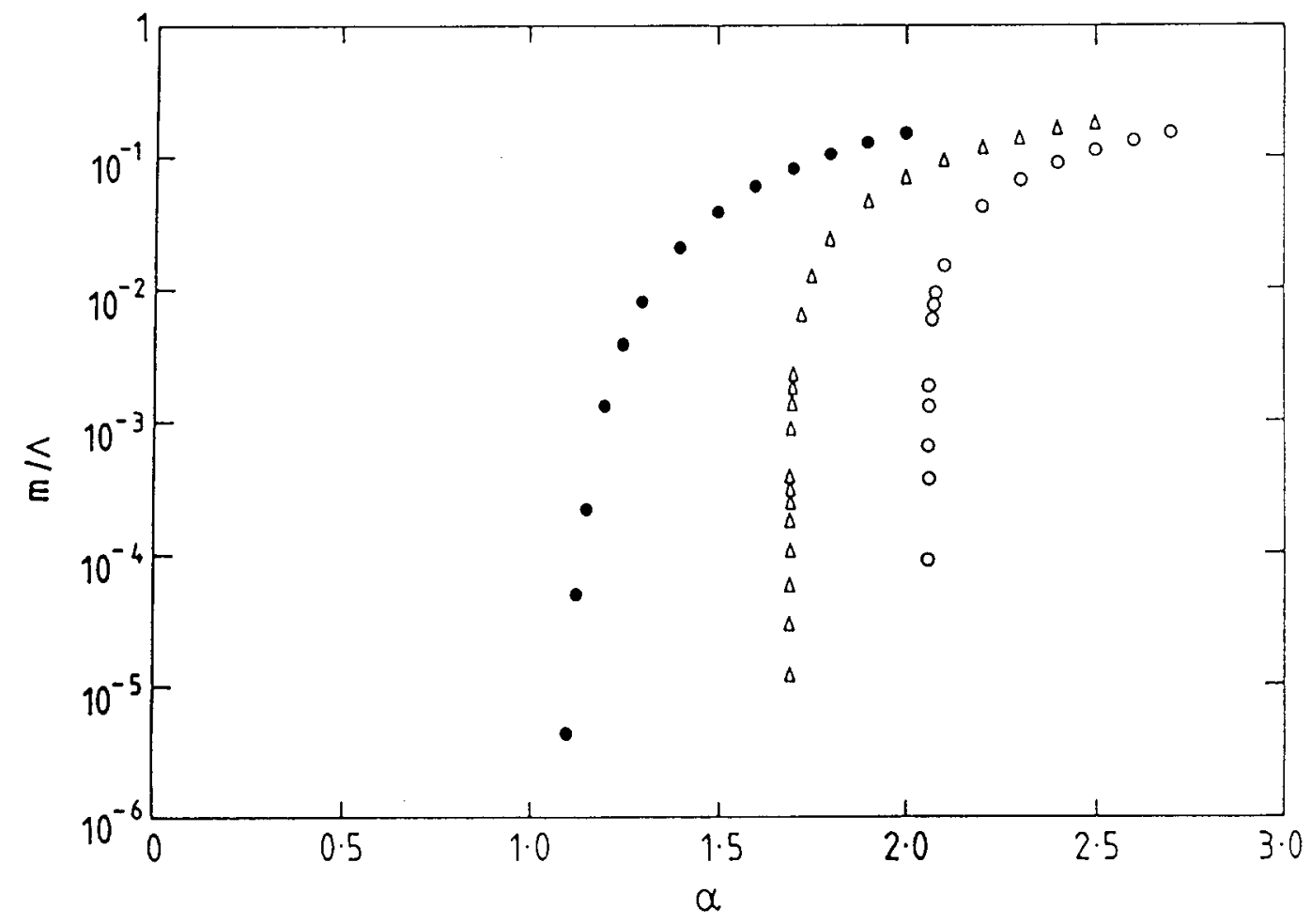

FIG. 4. Euclidean mass, $M=\mathcal{M}\left(M^{2}\right)$ dynamically generated in the rainbow approximation as a function of the coupling $\alpha$ in three different gauges: Landau $(\xi=0) \bullet$, Feynman $(\xi=1)$ $\triangle$, and Yennie $(\xi=3) \circ$ gauges.

Note that $\mathcal{M}=0$ is always a solution to Eq. (5). However, beyond the critical value of the coupling, the non-zero solution bifurcates away from the trivial solution. This is in complete contrast with the perturbation theory, where, even if we perform an all orders resummation using the Renormalization Group Equation, we end up 
with a result of the following form,

$$
\begin{aligned}
\mathcal{M}\left(p^{2}\right) & =m_{0} X\left(p^{2}\right) \\
X\left(p^{2}\right) & =\sum_{n}^{\infty} \sum_{m}^{n} \alpha^{n} A_{n} B_{m, n} \ln ^{m}\left(p^{2} / \Lambda^{2}\right)
\end{aligned}
$$

and the field remains massless to all orders if we start with a zero bare mass, $m_{0}=0$.

In contrast, non-perturbative dynamics is able to generate masses for particles even if they have zero bare mass. However, there are problems. As the critical coupling corresponds to a change of phase, we expect it to be independent of the gauge parameter. But when one solves the Eqs. (4) and (5) for different gauges, one finds that this is not the case, as depicted in Fig. 4. However, it is not difficult to trace the root of this problem. The full vertex of Eq. (1) has to satisfy the Ward-Takahashi identity for the fermion propagator to ensure its gauge covariance. However, the bare vertex that was used in Eq. (3) does not obey this identity. Therefore, one should not expect physical outputs to be gauge independent when the input is not.

\section{THE VERTEX}

We expect that any reasonable ansatz for the vertex should fulfill the following requirements:

- It must satisfy the Ward-Takahashi Identity in all gauges.

$$
q^{\mu} \Gamma_{\mu}=S_{F}^{-1}(k)-S_{F}^{-1}(p)
$$

- It must ensure that the fermion propagator of Eq. (1) is multiplicatively renormalizable.

- It must result in a critical coupling, at which mass is generated dynamically, that is gauge independent.

- It must be free of any kinematic singularities, i.e. it should have a unique limit when $k^{2} \rightarrow p^{2}$.

- It must have the same transformation properties as the bare vertex $\gamma^{\mu}$ under $C$ and $P$. 
Keeping in mind the form of the Ward-Takahashi identity, one can split the full vertex into two components, longitudinal and transverse:

$$
\Gamma^{\mu}(k, p)=\Gamma_{L}^{\mu}(k, p)+\Gamma_{T}^{\mu}(k, p)
$$

where, the transverse part of the vertex is defined by:

$$
q_{\mu} \Gamma_{T}^{\mu}(k, p)=0
$$

The Ward-Takahashi identity uniquely fixes the longitudinal part of the vertex, as shown by Ball and Chiu [4], to be

$$
\begin{aligned}
\Gamma_{L}^{\mu}(k, p) & =a\left(k^{2}, p^{2}\right) \gamma^{\mu}+b\left(k^{2}, p^{2}\right)(\not k+\not p)(k+p)^{\mu} \\
& -c\left(k^{2}, p^{2}\right)(k+p)^{\mu}
\end{aligned}
$$

where

$$
\begin{aligned}
& a\left(k^{2}, p^{2}\right)=\frac{1}{2}\left(\frac{1}{F\left(k^{2}\right)}+\frac{1}{F\left(p^{2}\right)}\right) \\
& b\left(k^{2}, p^{2}\right)=\frac{1}{2}\left(\frac{1}{F\left(k^{2}\right)}-\frac{1}{F\left(p^{2}\right)}\right) \frac{1}{k^{2}-p^{2}} \\
& c\left(k^{2}, p^{2}\right)=\left(\frac{\mathcal{M}\left(k^{2}\right)}{F\left(k^{2}\right)}-\frac{\mathcal{M}\left(p^{2}\right)}{F\left(p^{2}\right)}\right) \frac{1}{k^{2}-p^{2}}
\end{aligned}
$$

However, the transverse part remains arbitrary. Ball and Chiu [4] enumerated a basis of eight independent tensors in terms of which the most general form for the transverse part of the vertex can be written:

$$
\Gamma_{T}^{\mu}(k, p)=\sum_{i=1}^{8} \tau_{i}\left(k^{2}, p^{2}, q^{2}\right) T_{i}^{\mu}(k, p) .
$$

We list here only those four tensors which we shall need later:

$$
\begin{aligned}
& T_{2}^{\mu}(k, p)=\left(p^{\mu}(k . q)-k^{\mu}(p . q)\right)(\not k+\not p) \\
& T_{3}^{\mu}(k, p)=q^{2} \gamma^{\mu}-q^{\mu} \not 1 \\
& T_{6}^{\mu}(k, p)=\gamma^{\mu}\left(k^{2}-p^{2}\right)-(k+p)^{\mu}(\not k-\not p) \\
& T_{8}^{\mu}(k, p)=-\gamma^{\mu} p^{\nu} k^{\rho} \sigma_{\nu \rho}+p^{\mu} \not k-k^{\mu} \not p,
\end{aligned}
$$

with $\sigma_{\mu \nu}=\frac{1}{2}\left[\gamma_{\mu}, \gamma_{\nu}\right]$. The simplest choice is to take the transverse part to be zero. But Curtis and Pennington [5] showed that if we take the transverse part of the vertex 
to be zero, the fermion propagator is no longer multiplicatively renormalizable. They suggested the following transverse part of the vertex satisfying this requirement.

$$
\Gamma_{T}^{\mu}(k, p)=\frac{1}{2}\left(\frac{1}{F\left(k^{2}\right)}-\frac{1}{F\left(p^{2}\right)}\right) \frac{1}{d\left(k^{2}, p^{2}\right)} T_{6}^{\mu}(k, p),
$$

where, $d\left(k^{2}, p^{2}\right)=k^{2}$ for $k^{2} \gg p^{2} . d\left(k^{2}, p^{2}\right)$ must be symmetric in $k$ and $p$ and free of kinematic singularities leading to the proposal:

$$
d\left(k^{2}, p^{2}\right)=\frac{\left(k^{2}-p^{2}\right)^{2}+\left[\mathcal{M}^{2}\left(k^{2}\right)+\mathcal{M}^{2}\left(p^{2}\right)\right]^{2}}{k^{2}+p^{2}} .
$$

The vertex specified by Eqs. (8-12) will be referred to as the CP-vertex [5]. Curtis and Pennington solved the coupled equations for $F$ and $\mathcal{M}$ from Eq. (1), using this ansatz. They found that the gauge-dependence of the critical coupling at which the non-perturbative behaviour bifurcates away from the perturbative one reduces considerably, as seen by comparing Figs. 4 and 5 .

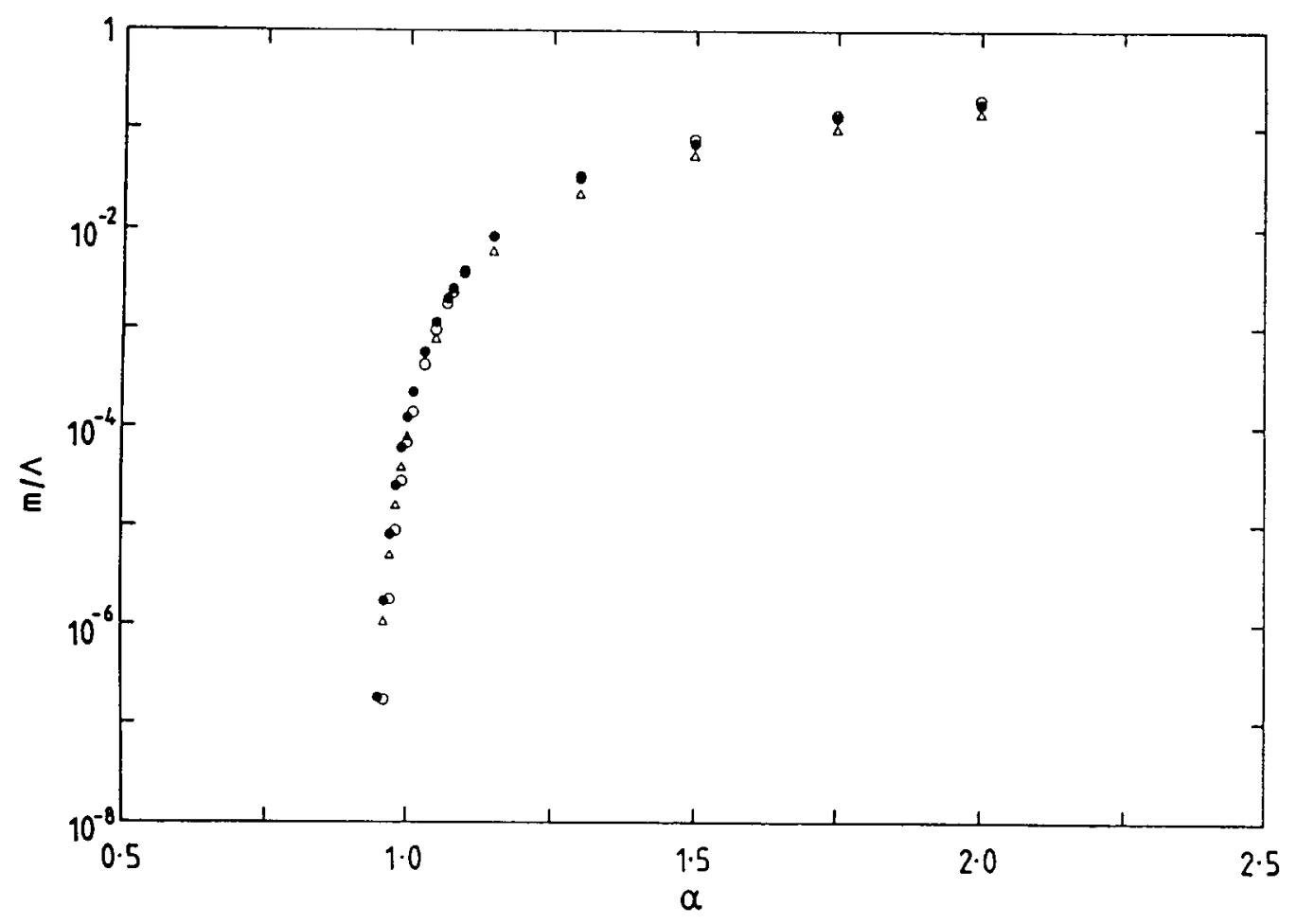

FIG. 5. Euclidean mass, $M=\mathcal{M}\left(M^{2}\right)$ dynamically generated using the CP-vertex as a function of the coupling $\alpha$ in three different gauges: Landau $(\xi=0) \bullet$, Feynman $(\xi=1) \triangle$, and Yennie $(\xi=3) \circ$ gauges. This plot is to be compared with the rainbow approximation results of Fig. 4. 


\section{BIFURCATION ANALYSIS}

To see this, Atkinson et al. [6] recently suggested a bifurcation analysis to study the phase change near the critical coupling. This is a precise way to locate the critical coupling as compared to the previous methods which rely on numerical calculations. This method amounts, in practice, simply to throwing away all terms that are quadratic or higher in the mass-function $\mathcal{M}$. Employing this procedure, and using the fact that at the critical coupling, $\mathcal{M}\left(p^{2}\right) \sim\left(p^{2}\right)^{-s}$ and $F\left(p^{2}\right) \sim\left(p^{2}\right)^{\nu}$ in Eq. (1), one arrives at the following equation in an arbitrary gauge:

$$
\begin{aligned}
\nu & =\frac{\alpha \xi}{4 \pi} \\
\xi & =\frac{3 \nu(\nu-s+1)}{2(1-s)}[3-\pi \cot \pi(\nu-s)+2 \pi \cot \pi s-\pi \cot \pi \nu \\
& \left.+\frac{1}{\nu}+\frac{1}{\nu+1}+\frac{1}{\nu}+\frac{2}{1-s}+\frac{3}{s-\nu}+\frac{1}{s-\nu-1}\right] .
\end{aligned}
$$

There are two roots of this latter equation for $s$ between 0 and 1. Bifurcation occurs

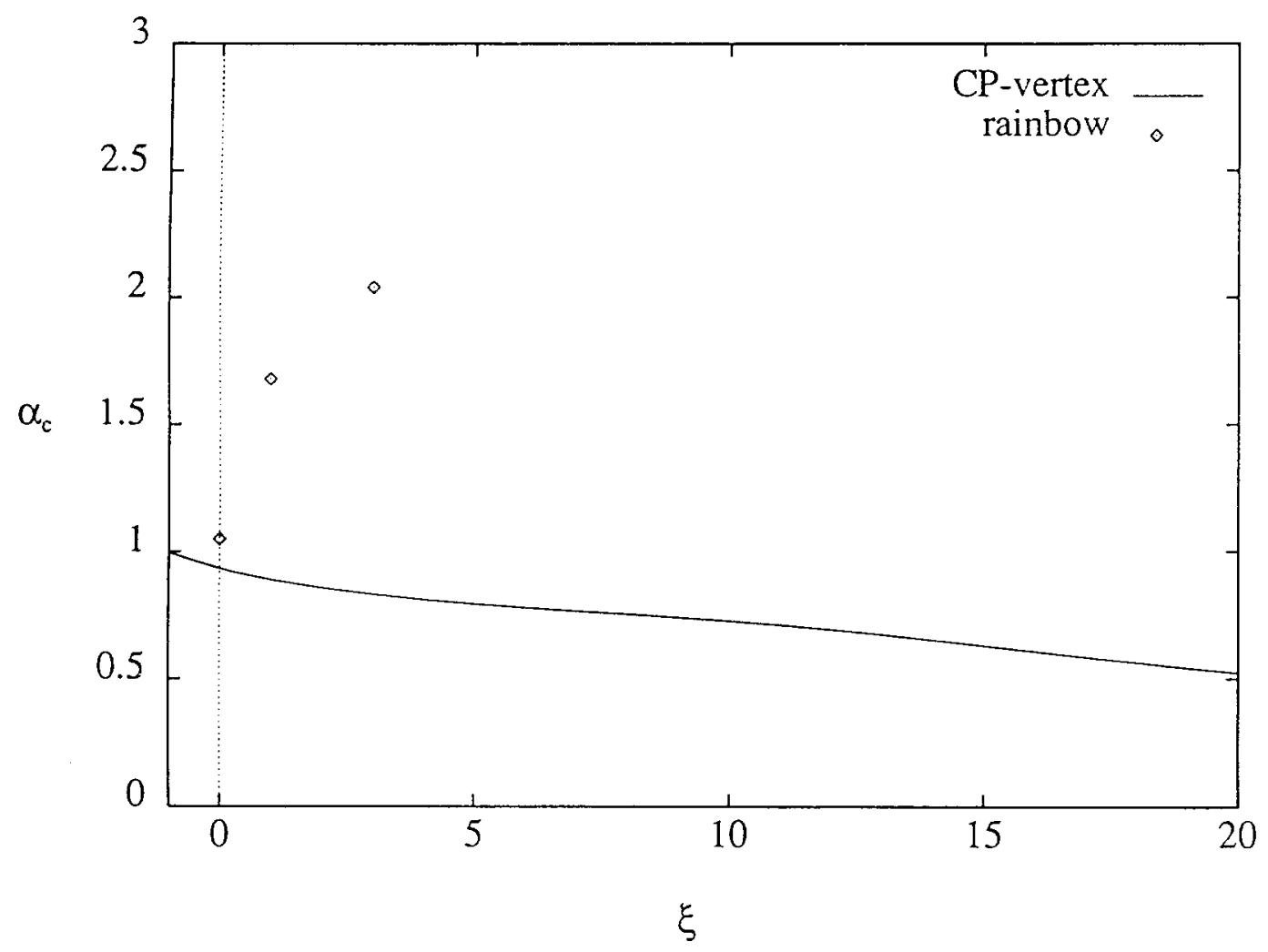

FIG. 6. Critical coupling, $\alpha_{c}$, as a function of the gauge parameter, $\xi$ (solid line). The corresponding values for the rainbow approximation have also been shown $\diamond$. 
The bifurcation point defines the critical coupling, $\alpha_{c}$. Numerically, $\alpha_{c}=0.933667$ in the Landau gauge. For each value of the gauge parameter, these equations can be solved for $\nu, s_{c}$ and $\alpha_{c}$. The solution found by Atkinson et al [6] is displayed in Fig. 6. For comparison, the points for the bare vertex have also been shown. One can see that the gauge dependence has considerably been reduced, as was seen earlier. However weak this variation, any gauge dependence shows that the $C P$ vertex cannot be the exact choice.

\section{CONSTRAINTS OF MULTIPLICATIVE RENORMALIZABILITY}

To find a vertex that ensures the gauge independence of the critical coupling, we start off by making three assumptions. Firstly, we demand that a chirally-symmetric solution should be possible when the bare mass is zero, just as in perturbation theory. This is most easily accomplished if the sum in Eq. (9) involves just $i=2,3,6$ and 8. The second assumption is that the functions, $\tau_{i}$, multiplying the transverse vectors, Eq. (9), only depend on $k^{2}$ and $p^{2}$, but not $q^{2}$. The third assumption is that the transverse part of the vertex vanishes in the Landau gauge. The motivation for this comes from the lowest order perturbative calculation for the transverse vertex, satisfied by Eq. (11). These conditions fix the $\tau_{i}$ of Eq. (9). Multiplicative renormalizability of the wavefunction renormalization $F\left(p^{2}\right)$ enables us to write $\tau_{6}$ and $\bar{\tau}$ in terms of one function $W_{1}(x)[7]$ :

$$
\begin{aligned}
\bar{\tau}\left(k^{2}, p^{2}\right)= & \frac{1}{4} \frac{1}{k^{2}-p^{2}} \frac{1}{s_{1}\left(k^{2}, p^{2}\right)}\left[W_{1}\left(\frac{k^{2}}{p^{2}}\right)-W_{1}\left(\frac{p^{2}}{k^{2}}\right)\right], \\
\tau_{6}\left(k^{2}, p^{2}\right)= & -\frac{1}{2} \frac{k^{2}+p^{2}}{\left(k^{2}-p^{2}\right)^{2}}\left(\frac{1}{F\left(k^{2}\right)}-\frac{1}{F\left(p^{2}\right)}\right)+\frac{1}{3} \frac{k^{2}+p^{2}}{k^{2}-p^{2}} \bar{\tau}\left(k^{2}, p^{2}\right) \\
& +\frac{1}{6} \frac{1}{k^{2}-p^{2}} \frac{1}{s_{1}\left(k^{2}, p^{2}\right)}\left[W_{1}\left(\frac{k^{2}}{p^{2}}\right)+W_{1}\left(\frac{p^{2}}{k^{2}}\right)\right],
\end{aligned}
$$

where $\bar{\tau}$ is the combination of $\tau_{i}$ given by:

$$
\bar{\tau}\left(k^{2}, p^{2}\right)=\tau_{3}\left(k^{2}, p^{2}\right)+\tau_{8}\left(k^{2}, p^{2}\right)-\frac{1}{2}\left(k^{2}+p^{2}\right) \tau_{2}\left(k^{2}, p^{2}\right)
$$

and

$$
s_{1}\left(k^{2}, p^{2}\right)=\frac{k^{2}}{p^{2}} F\left(k^{2}\right)+\frac{p^{2}}{k^{2}} F\left(p^{2}\right)
$$


The condition of multiplicative renormalizability, i.e, $F\left(p^{2}\right) \sim\left(p^{2}\right)^{\nu}$, constrains the otherwise arbitrary function $W_{1}$ as follows:

$$
\int_{0}^{1} d x W_{1}(x)=0
$$

It should be noted that, with the simplest choice $W_{1}=0$, the massless CP-vertex, Eqs. (11,12) emerges.

\section{CONSTRAINTS OF GAUGE INVARIANCE}

At the bifurcation point, as stated before, multiplicative renormalizability forces a simple power behaviour for the mass function as well as for the wavefunction renormalization. Such a multiplicatively renormalizable mass function must exist in all gauges. Consequently, the exponent, $s_{c}$, must be gauge independent. Moreover, dynamical mass generation marks a physical phase change and so the critical coupling, $\alpha_{c}$, must also be gauge independent. Thus the critical values, $\alpha_{c}, s_{c}$, found in the Landau gauge must hold in all gauges. Using this physically motivated argument, the equation for the mass function gives $\tau_{2}, \tau_{3}$ and $\tau_{8}$ in terms of a function $W_{2}(x)$ [8]:

$$
\begin{aligned}
\tau_{2}\left(k^{2}, p^{2}\right)= & \frac{2 \xi}{\left(k^{2}-p^{2}\right)^{2}} \frac{q_{2}\left(k^{2}, p^{2}\right)}{s_{2}\left(k^{2}, p^{2}\right)}-6 \frac{\tau_{6}\left(k^{2}, p^{2}\right)}{\left(k^{2}-p^{2}\right)} \\
& -\frac{1}{\left(k^{2}-p^{2}\right)^{2}} \frac{1}{s_{2}\left(k^{2}, p^{2}\right)}\left[W_{2}\left(\frac{k^{2}}{p^{2}}\right)+W_{2}\left(\frac{p^{2}}{k^{2}}\right)\right] \\
& -\frac{k^{2}+p^{2}}{\left(k^{2}-p^{2}\right)^{3}} \frac{1}{s_{2}\left(k^{2}, p^{2}\right)}\left[W_{2}\left(\frac{k^{2}}{p^{2}}\right)-W_{2}\left(\frac{p^{2}}{k^{2}}\right)\right], \\
\tau_{3}\left(k^{2}, p^{2}\right)= & -\frac{k^{2}+p^{2}}{k^{2}-p^{2}} \tau_{6}\left(k^{2}, p^{2}\right) \\
& +\frac{1}{k^{2}-p^{2}} \frac{1}{s_{2}\left(k^{2}, p^{2}\right)}\left[\frac{1}{2} r_{2}\left(\frac{k^{2}}{p^{2}}\right)-\frac{\xi}{3} q_{3}\left(k^{2}, p^{2}\right)\right] \\
& -\frac{1}{6} \frac{k^{2}+p^{2}}{\left(k^{2}-p^{2}\right)^{2}} \frac{1}{s_{2}\left(k^{2}, p^{2}\right)}\left[W_{2}\left(\frac{k^{2}}{p^{2}}\right)+W_{2}\left(\frac{p^{2}}{k^{2}}\right)\right] \\
& +\frac{1}{6} \frac{k^{4}+p^{4}-6 k^{2} p^{2}}{\left(k^{2}-p^{2}\right)^{3}} \frac{1}{s_{2}\left(k^{2}, p^{2}\right)}\left[W_{2}\left(\frac{k^{2}}{p^{2}}\right)-W_{2}\left(\frac{p^{2}}{k^{2}}\right)\right],
\end{aligned}
$$

and 


$$
\begin{aligned}
\tau_{8}\left(k^{2}, p^{2}\right)= & -2 \frac{k^{2}+p^{2}}{k^{2}-p^{2}} \tau_{6}\left(k^{2}, p^{2}\right)+\bar{\tau}\left(k^{2}, p^{2}\right) \\
& -\frac{1}{k^{2}-p^{2}} \frac{1}{s_{2}\left(k^{2}, p^{2}\right)}\left[\frac{1}{2} r_{2}\left(\frac{k^{2}}{p^{2}}\right)-\frac{\xi}{3} q_{8}\left(k^{2}, p^{2}\right)\right] \\
& -\frac{1}{3} \frac{k^{2}+p^{2}}{\left(k^{2}-p^{2}\right)^{2}} \frac{1}{s_{2}\left(k^{2}, p^{2}\right)}\left[W_{2}\left(\frac{k^{2}}{p^{2}}\right)+W_{2}\left(\frac{p^{2}}{k^{2}}\right)\right] \\
& -\frac{2}{3} \frac{k^{4}+p^{4}}{\left(k^{2}-p^{2}\right)^{3}} \frac{1}{s_{2}\left(k^{2}, p^{2}\right)}\left[W_{2}\left(\frac{k^{2}}{p^{2}}\right)-W_{2}\left(\frac{p^{2}}{k^{2}}\right)\right],
\end{aligned}
$$

where

$$
\begin{aligned}
r_{1}\left(\frac{k^{2}}{p^{2}}\right) & =\left(\frac{k^{2}}{p^{2}}\right)\left[1-\left(\frac{k^{2}}{p^{2}}\right)^{\nu}\right]-\left(\frac{k^{2}}{p^{2}}\right)^{-1}\left[1-\left(\frac{k^{2}}{p^{2}}\right)^{-\nu}\right], \\
r_{2}\left(\frac{k^{2}}{p^{2}}\right) & =\left(\frac{k^{2}}{p^{2}}\right)^{\frac{1}{2}-s_{c}}\left[1-\left(\frac{k^{2}}{p^{2}}\right)^{\nu}\right]-\left(\frac{k^{2}}{p^{2}}\right)^{s_{c}-\frac{1}{2}}\left[1-\left(\frac{k^{2}}{p^{2}}\right)^{-\nu}\right], \\
s_{1}\left(k^{2}, p^{2}\right) & =\frac{k^{2}}{p^{2}} F\left(k^{2}\right)+\frac{p^{2}}{k^{2}} F\left(p^{2}\right), \\
s_{2}\left(k^{2}, p^{2}\right) & =\frac{k}{p} \frac{\mathcal{M}\left(k^{2}\right)}{\mathcal{M}\left(p^{2}\right)} F\left(k^{2}\right)+\frac{p}{k} \frac{\mathcal{M}\left(p^{2}\right)}{\mathcal{M}\left(k^{2}\right)} F\left(p^{2}\right), \\
q_{2}\left(k^{2}, p^{2}\right) & =\frac{1}{k^{2}-p^{2}}\left[\frac{k^{3}}{p} \frac{\mathcal{M}\left(k^{2}\right) F\left(k^{2}\right)}{\mathcal{M}\left(p^{2}\right) F\left(p^{2}\right)}-\frac{p^{3}}{k} \frac{\mathcal{M}\left(p^{2}\right) F\left(p^{2}\right)}{\mathcal{M}\left(k^{2}\right) F\left(k^{2}\right)}\right], \\
q_{3}\left(k^{2}, p^{2}\right) & =\frac{k p}{\left(k^{2}-p^{2}\right)^{2}}\left[\left(p^{2}-3 k^{2}\right) \frac{\mathcal{M}\left(k^{2}\right) F\left(k^{2}\right)}{\mathcal{M}\left(p^{2}\right) F\left(p^{2}\right)}-\left(k^{2}-3 p^{2}\right) \frac{\mathcal{M}\left(k^{2}\right) F\left(k^{2}\right)}{\mathcal{M}\left(p^{2}\right) F\left(p^{2}\right)}\right], \\
q_{8}\left(k^{2}, p^{2}\right) & =\frac{1}{\left(k^{2}-p^{2}\right)^{2}}\left[\frac{k}{p}\left(3 k^{4}+p^{4}\right) \frac{\mathcal{M}\left(k^{2}\right) F\left(k^{2}\right)}{\mathcal{M}\left(p^{2}\right) F\left(p^{2}\right)}-\frac{p}{k}\left(k^{4}+3 p^{4}\right) \frac{\mathcal{M}\left(k^{2}\right) F\left(k^{2}\right)}{\mathcal{M}\left(p^{2}\right) F\left(p^{2}\right)}\right] .
\end{aligned}
$$

and the function $W_{2}$ is constrained, by the gauge invariance of the mass function and the critical coupling, to obey the following integral equation,

$$
\int_{0}^{1} \frac{d x}{\sqrt{x}} W_{2}(x)=0 \quad,
$$

at the critical coupling $\alpha=\alpha_{c}$. In order to make sure that none of the functions $\tau_{i}$ has kinematic singularities as $k^{2} \rightarrow p^{2}, W_{1}$ and $W_{2}$ should also satisfy the following conditions:

$$
\begin{aligned}
W_{1}(1)+W_{1}^{\prime}(1) & =-6 \nu, \\
W_{2}(1)+2 W_{2}^{\prime}(1) & =2 \xi(\nu-s-1) .
\end{aligned}
$$

This defines the construction of the full vertex via Eqs. (6-10) [8]. 


\section{CONCLUSIONS}

Above we have presented a truncation of the fermion Schwinger-Dyson equation for the quenched QED, which respects the key properties of the theory. We have constructed a non-perturbative vertex in terms of the constrained functions $W_{i}(x)(i=1,2)$. It satisfies the Ward-Takahashi identity, ensures the fermion propagator is multiplicatively renormalizable, agrees with one loop perturbation theory for large momenta and enforces a gauge independent chiral symmetry breaking phase transition. This study motivates the need for a realistic investigation of $t \bar{t}$ condensates as the source of the electroweak symmetry breaking. Including the four fermion interaction, the Dyson-Schwinger equation for the fermion propagator becomes:

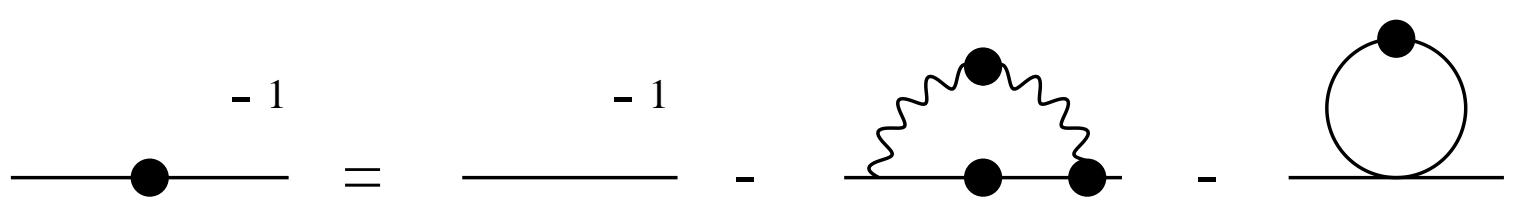

FIG. 7. Dyson-Schwinger equation for the fermion propagator, including the four fermion interaction term.

We need to solve this equation in a gauge invariant way. The study of quenched QED presented here suggests that a proper choice of the vertex can guarantee the gauge independence of the physical observables. However, a realistic calculation, of course, requires the unquenching of the theory which complicates the problem significantly. The fermion-boson vertex (in particular its transverse part) will intimately depend on the photon renormalization function in a non-perturbative way not yet understood. The discussion for quenched QED presented here provides the starting point for such an investigation of full QED.

\section{ACKNOWLEDGEMENTS}

This work was performed in collaboration with M.R. Pennington. I wish to thank the Government of Pakistan for a research studentship and the University of Durham and Institut d'Etudes Scientfiques de Cargèse for providing me with the funds to attend the School. 


\section{REFERENCES}

[1] W.A. Bardeen, C.T. Hill and M. Lindner, Phys. Rev. D41 1647 (1990).

[2] V.A. Miransky, Nuovo Cim. 90A 149 (1985) ;

Sov. Phys. JETP 61905 (1985);

P.I. Fomin, V.P. Gusynin, V.A. Miransky and Yu.A. Sitenko,

La rivista del Nuovo Cim. 6, numero 5, 1 (1983).

[3] D.C. Curtis and M.R. Pennington, Phys. Rev. D48 4933 (1993).

[4] J.S. Ball and T.W. Chiu, Phys. Rev. D22 2542 (1980).

[5] D.C. Curtis and M.R. Pennington, Phys. Rev. D42 4165 (1990).

[6] D. Atkinson, J.C.R. Bloch, V.P. Gusynin, M.R. Pennington and M. Reenders, Phys. Lett. B329 117 (1994).

[7] Z. Dong, H.J. Munczek and C.D. Roberts, preprint ANL-PHY-7711-94.

[8] A. Bashir and M.Pennington, "Gauge Independent Chiral Symmetry Breaking in Quenched QED ", University of Durham preprint DTP-94/48 (June, 94) Phys. Rev. (to be published). 